\title{
Dynamics and statistics of heavy particles in turbulent flows
} M. CENCINI ${ }^{1}$, J. BEC ${ }^{2}$, L. BIFERALE ${ }^{3}$, G. BOFFETTA ${ }^{4}$, A. CELANI ${ }^{5}$, A. S. LANOTTE 6 , S. MUSACCHIO ${ }^{5}$ and F. TOSCHI ${ }^{7}$

${ }^{1}$ SMC-INFM c/o Dept. of Physics University of Rome "La Sapienza", and CNR-ISC via dei Taurini, 19 I-00185 Roma, Italy

${ }^{2}$ CNRS, Lab. Cassiopée, OCA, B.P. 4229, 06304 Nice Cedex 4, France

${ }^{3}$ Dept. of Physics and INFN, University of Rome "Tor Vergata", ${ }^{4}$ Dept. of Physics and INFN, University of Torino,

${ }^{5}$ CNRS, INLN, 1361 Route des Lucioles, F-06560 Valbonne, France

${ }^{6}$ CNR-ISAC, Sezione di Lecce, Str. Prov. Lecce-Monteroni, I-73100 Lecce, Italy

${ }^{7}$ CNR-IAC, Viale del Policlinico 137, I-00161 Roma, Italy and INFN, Sezione di Ferrara, via G. Saragat 1, I-44100, Ferrara, Italy.

\begin{abstract}
We present the results of Direct Numerical Simulations (DNS) of turbulent flows seeded with millions of passive inertial particles. The maximum Reynolds number is $R e_{\lambda} \sim 200$. We consider particles much heavier than the carrier flow in the limit when the Stokes drag force dominates their dynamical evolution. We discuss both the transient and the stationary regimes. In the transient regime, we study the growth of inhomogeneities in the particle spatial distribution driven by the preferential concentration out of intense vortex filaments. In the stationary regime, we study the acceleration fluctuations as a function of the Stokes number in the range $S t \in[0.16: 3.3]$. We also compare our results with those of pure fluid tracers $(S t=0)$ and we find a critical behavior of inertia for small Stokes values. Starting from the pure monodisperse statistics we also characterize polydisperse suspensions with a given mean Stokes, $\overline{S t}$.
\end{abstract}

\section{INTRODUCTION}

Suspensions of dust, impurities, droplets, bubbles, and other finite-size particles advected by incompressible turbulent flows are commonly encountered in many natural phenomena and industrial processes. These finite-size particles, whose density may differ from that of the underlying fluid, cannot be modeled as point-like tracers because of their inertia. Often, they are characterized by the presence of strong inhomogeneities in their spatial distribution. Indeed, light (heavy) particles tend to concentrate into specific regions of the flow characterized by high (low) values of the vorticity. Such a phenomenon is dubbed 'preferential concentration' [1]. The inhomogeneities then appearing in the particle spatial distribution are important because they affect the probability to find close particle pairs and thus influence their possibility to collide, or to have biological and chemical interactions. Examples showing the importance of this phenomenon are the formation of rain drops by coalescence in warm clouds [2, 3, 4, 5], or the coexistence between several species of plankton in the hydrosphere [6, 7]. Engineering applications encompass optimization of spray combustion in diesel engines [8] and in rocket propellers 9 |. Inertial particles are also important for the problem of dispersion of dust, chemicals or aerosols in the atmosphere [10, 11].

Recently, much effort have been devoted to the study of phenomena related to preferential concentration of inertial particles in turbulent flows by means of both theoretical 12, 13, 14, 15] and computational [16, 17, 18, 19, 20, 21] approaches. Progresses in the characterization of the statistical features of particle clusters have been achieved by studying inertial particles evolving in laminar stochastic flows [12, 22, 23, 24, 25] and two dimensional turbulent flows [26]. Experimental results are reviewed in Ref. 1]. Recently, experiments [27] have been also realized borrowing techniques from Lagrangian turbulence [28, 29, 30, 31, 32].

Considerable less attention has been paid to other aspects of inertial particle dynamics, and in particular to the statistical properties of their acceleration in turbulent flows. This is an important issue relevant, for example, to the development of stochastic models of particle motion [33]. It is also interesting to contrast the statistics of the acceleration of inertial particles with that of fluid tracers which has been extensively studied by means of experiments 28, 29, 30, 31, 34], numerical simulations [35, 36, 37, 38, 39, 40] and theoretical approaches [36, 41, 42. This is even more interesting on the light of recent experiments [27] designed to study inertial particles in turbulent flows by means of Lagrangian measurements. Concerning fluid tracers, it has been found that the acceleration probability density function (pdf) displays the typical fat tails of highly intermittent signals [28, 32]. The tails of the pdf have been associated to trapping events into vortex filaments [28, 35]. Moreover, a model based on the multifractal description of turbulence has been proved able to describe the pdf of the acceleration tracers $\underline{36}$. Similar questions are still open for inertial particles. Because of the centrifugal force acting on such particles, inertia is expected to considerably change the statistics of trapping events into vortex filaments. It is important to quantify this effect by looking at the 
modification of the pdf tails as a function of the degree of inertia. Moreover, it is not known how to generalize the multifractal approach used for passive tracers to the case of passive particles with inertia.

In this paper we focus on the statistical properties of particles much heavier than the carrier fluid. We are interested in investigating, by means of high-resolution Direct Numerical Simulations (DNS), the combined effects of preferential concentration and of inertia in determining the statistics of acceleration. With this aim, we review and extend the results presented in Ref. [43]. In particular, we perform a systematic study as a function of the Stokes number. Here Stokes is defined as $S t=\tau_{s} / \tau_{\eta}$, being $\tau_{s}$ the typical response time of the particle and $\tau_{\eta}$ the Kolmogorov time of the flow.

Most results concern monodisperse suspensions, i.e. set of particles with a unique Stokes number. Since laboratory and natural suspensions are typically polydisperse, we shall extend our analysis to this case also.

Understanding the relevant time scales to reach a stationary regime is important both for experimental studies - often limited in running time - and for a dynamical description of preferential concentration. We present an analysis of the transient regime, i.e. the time window necessary for the particles to reach a statistically steady regime, starting from their initial configuration.

The paper is organized as follows. In Sec. III we recall the equations of motion for the case of inertial particles much heavier than the surrounding fluid. A detailed description of the numerical set up is given in Sec. III In Sec. IV the validity of the model equations for our numerical experiments is considered. Moreover, we discuss the statistical features of preferential concentration both during the transient and in the statistically stationary regime. The most relevant features of particle acceleration statistics for monodisperse suspensions are discussed in Sec. $\nabla$ Section $\nabla \mathbf{D I}$ is devoted to extend the previous analysis to polydisperse cases. Conclusions and perspectives are drawn in Sec. VII

\section{HEAVY PARTICLE DYNAMICS}

The equations of motion of a small, rigid, spherical particle immersed in an incompressible flow have been consistently derived from first principles in Refs. [44, 45, 46] under a certain number of assumptions. The main working hypothesis is to consider low particle concentration, so that it is possible to neglect collisions, particle-to-particle hydrodynamic interactions and the feedback of particles on the carrier fluid. Another hypothesis is that the particle radius $r$ is much smaller than any active scale of the turbulent flow, namely that $r \ll \eta$, where $\eta$ denotes the Kolmogorov dissipative scale. Finally, the particle Reynolds number $\operatorname{Re}_{p}=r|V-u| / \nu$, where $|V-u|$ is the typical relative velocity of the particle with the flow and $\nu$ is the fluid kinematic viscosity, has to be small, i.e. $R e_{p} \ll 1$. These hypotheses lead to describe the fluid flow surrounding the particle as a Stokes flow and to write a close differential equation for the motion of the particle [47]. The dynamics of a single particle then depends on only two dimension-less parameters. The first is $\beta=3 \rho_{f} /\left(\rho_{f}+2 \rho_{p}\right)$, accounting for the mass density ratio between the particle, $\rho_{p}$, and the fluid, $\rho_{f}$. The second control parameter is the Stokes number $S t=\tau_{s} / \tau_{\eta}$, where the particle response time is $\tau_{s}=r^{2} /(3 \beta \nu)$. Further, neglecting gravity, and assuming that the particle is much heavier than the fluid, the particle velocity is to leading order a solution to [12, 45]:

$$
\frac{d V}{d t}=\beta D_{t} u(X, t)-\frac{V(t)-u(X(t), t)}{\tau_{s}} .
$$

where $X$ denotes the particle position, $u(x, t)$ the fluid velocity field, and $D_{t} u(X, t)=\partial_{t} u+u \cdot \nabla u$ is the fluid acceleration at the particle position. As long as $D_{t} u(X, t)$ does not become too large (see Sect. IV A), the first term in the right-hand side of (11) can be neglected when $\beta \ll 1$, so that the equations can be further simplified to

$$
\begin{aligned}
& \frac{d X}{d t}=V(t) \\
& \frac{d V}{d t}=-\frac{1}{\tau_{s}}(V(t)-u(X(t), t)) .
\end{aligned}
$$

It is interesting to compare the evolution equations for particles (2) with that for the motion of fluid tracers

$$
\frac{d x(t)}{d t}=u(x(t), t)
$$

which corresponds to the limit $\tau_{s}=0$ of (2). Tracers may be seen as $S t=0$ particles. 


\section{DETAILS ON THE DNS AND THE DATA SET}

The carrier fluid is evolved according to the incompressible Navier-Stokes equations

$$
\frac{\partial u}{\partial t}+u \cdot \nabla u=-\frac{\nabla p}{\rho_{f}}+\nu \Delta u+f
$$

where $p$ is the pressure field and $f$ is the external energy source, $\langle f \cdot u\rangle=\epsilon$. These are solved on a cubic grid with periodic boundary conditions. Energy is injected by keeping constant the spectral content of the two smallest wavenumber shells [4]. The viscosity is chosen so to have a well resolved dissipative range, $\eta \approx \Delta x$ where $\Delta x$ is the grid spacing. We use a fully dealiased pseudospectral algorithm with $2^{\text {nd }}$ order Adam-Bashforth time stepping. We performed three sets of runs at resolution $N^{3}$ with $N=128,256,512$, the corresponding Reynolds numbers are $R_{\lambda}=65,105,185$ (see also Ref. [43]).

Particle dynamics, Eqn. (2), is integrated in parallel with that of the fluid by means of a $2^{\text {nd }}$ order Adam-Bashforth time-stepping, in which the fluid velocity at particle position is estimated by linear interpolation.

For each set of runs, the following procedure has been adopted. We integrate the Navier-Stokes equations until the flow reaches a statistically steady state. Once a turbulent configuration for the flow is obtained, millions of particles and tracers are seeded in the flow. Their initial positions are uniformly distributed in the volume, and velocities are chosen equal to the local fluid velocity. Equations (2) for particles and (3) for tracers are then advanced in parallel with those for the fluid (4). We consider particles with 15 different Stokes numbers ranging from 0.16 to 3.5 (see caption of Table $\llbracket$ for the exact values), and a set of tracers, $S t=0$. For each set of particles with a given Stokes number, we save the position and the velocity of $N_{t}$ particles every $d t=\tau_{\eta} / 10$, with a maximum number of recorded trajectories of $N_{t}=5 \cdot 10^{5}$ for the highest resolution. Along these trajectories we also store the velocity of the carrier fluid. At a lower frequency, $\sim 10 \tau_{\eta}$, we save the position and velocity of a larger number $N_{p}$ of particles $\left(\right.$ up to $7.5 \cdot 10^{6}$ per $S t$, at the highest resolution) together with the Eulerian velocity field.

A summary of the physical parameters for the run at the highest resolution, the one discussed in this paper, is given in Table 1

\begin{tabular}{|cccccccc|}
\hline$R_{\lambda}$ & $u_{\mathrm{rms}}$ & $\varepsilon$ & $\nu$ & $\eta$ & $L T_{E}$ & $\tau_{\eta}$ \\
\hline 185 & 1.4 & 0.94 & 0.00205 & 0.010 & $\pi$ & 2.2 & 0.047 \\
\hline \multicolumn{11}{|c}{} & & & & \\
\hline$T_{\text {tot }}$ & $T_{t r}$ & $\Delta x$ & $N^{3}$ & $N_{t}$ & $N_{p}$ & $N_{\text {tot }}$ \\
\hline 12 & 4 & 0.012 & $512^{3}$ & $5 \cdot 10^{5}$ & $7.5 \cdot 10^{6}$ & $12 \cdot 10^{7}$ \\
\hline
\end{tabular}

TABLE I: DNS parameters. Microscale Reynolds number $R_{\lambda}$, root-mean-square velocity $u_{\mathrm{rms}}$, energy dissipation $\varepsilon$, viscosity $\nu$, Kolmogorov length scale $\eta=\left(\nu^{3} / \varepsilon\right)^{1 / 4}$, integral scale $L$, large-scale eddy Eulerian turnover time $T_{E}=L / u_{\mathrm{rms}}, \mathrm{Kolmogorov}$ timescale $\tau_{\eta}=(\nu / \varepsilon)^{1 / 2}$, total integration time $T_{t o t}$, duration of the transient regime $T_{t r}$, grid spacing $\Delta x$, resolution $N^{3}$, number of trajectories of inertial particles for each Stokes $N_{t}$ saved at frequency $\tau_{\eta} / 10$, number of particles $N_{p}$ per Stokes stored at frequency $10 \tau_{\eta}$, total number of advected particles $N_{t o t}$. Errors on all statistically fluctuating quantities are of the order of $10 \%$. The Stokes number are $S t=(0 ; 0.16 ; 0.27 ; 0.37 ; 0.48 ; 0.59 ; 0.69 ; 0.80 ; 0.91 ; 1.01 ; 1.12 ; 1.34 ; 1.60 ; 2.03 ; 2.67 ; 3.31)$

\section{STATISTICAL ANALYSIS OF THE DATA SET}

First, we check if the approximate equations (2) are consistent with the assumptions used to derive them, in the range of parameters of our DNS. Second, we study how particles, injected homogeneously in the domain, reach a statistically inhomogeneous steady state spatial distribution. Further, we characterize preferential concentration of particles as a function of the Stokes number in the stationary regime.

\section{A. Limit of validity for the particle equations of motion}

To validate our working hypotheses, we check that they are self-consistent with neglecting the term proportional to $\beta$ in (1). In particular, we want to see what are the constraints on the values of $\beta$ and on the particle radius $r$ that should be satisfied in order to fulfill the assumption $\beta\left|D_{t} u\right| \ll|u-V| / \tau_{s}$.

The first observation is that from the definition of $S t$ and from the dimensional estimation of the Kolmogorov scale 


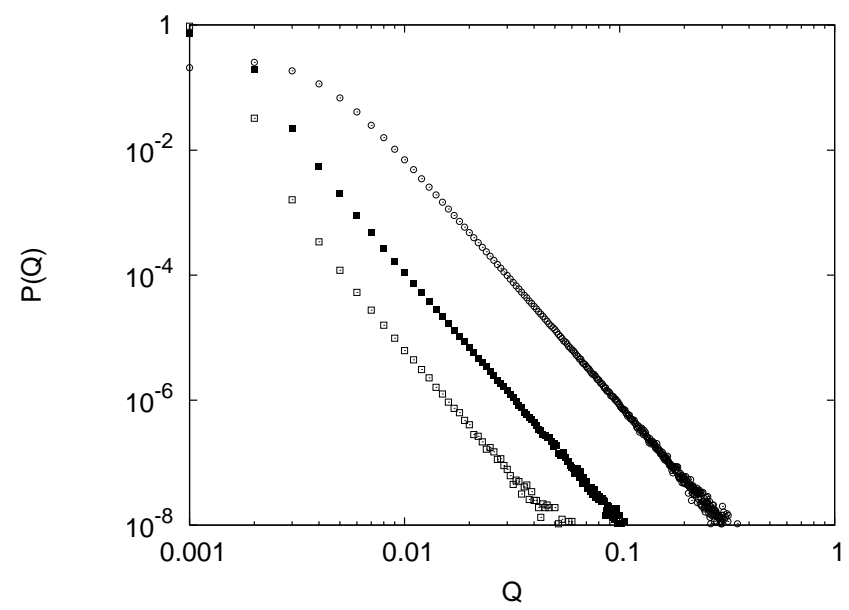

FIG. 1: $\log -\log$ plot of the pdf $P(Q)$ vs $Q=\beta \tau_{s}\left|d_{t} u\right| /|u-V|$, with $\beta=0.001$ and for three different Stokes numbers $S t=0.16,0.59,3.31$ from the inner to the outer curve, respectively.

$\eta=\left(\nu^{3} / \epsilon\right)^{1 / 4}$, one has $r / \eta=\sqrt{3 \beta S t}$, meaning that for $S t \ll 1$ there are no severe constraints on the value of $\beta$. Differently, for large $S t$, in order to have $r \ll \eta$, one may need to use unphysical small values of $\beta$. To see whether this is the case, let us consider a typical example of parameter values: the case of water in air $\beta \approx 0.001$, and $S t=3$ (which is the largest value used in our simulations). In the worst case, we have $r / \eta \leq 0.3$ which is still small enough to justify to neglect the Basset history and the added mass terms, and the Faxén corrections [45], leading to Eq. (11) for the particle dynamics. We now show that it is self-consistent to further neglect the term $\beta D_{t} u$. Since in the DNS we do not have stored the fluid acceleration at particle positions for the whole trajectory, we assume $D_{t} u \approx d_{t} u$, i.e. we estimate the fluid acceleration at the particle position with the time derivative of the fluid velocity along the particle path. The validity of this approximation relies on the fact that the pdf of $d_{t} u$ that, for large $S t$, are very close to those of $D_{t} u$ (not shown), meaning that strong acceleration events are well represented.

Figure 1 shows, for three representative Stokes numbers, the distribution of the ratio

$$
Q=\beta \frac{|d u / d t|}{|(u-V)| / \tau_{s}}
$$

As one can see, the statistical weight of events $Q>0.1$ is very low, meaning that with a very high probability the equations (21) are a valid approximation. Of course, for density ratios smaller than $\beta=0.001$ the approximation will work even better. If one considers for instance sand dust transported in the atmosphere at an altitude of roughly $5 \mathrm{~km}, \beta$ is reduced by a factor $1 / 10$ and in this case our simulations do not catch any event where $Q>0.1$.

We thus conclude that in the range of $\beta$ considered above, the fluid inertia term is also negligible and equations (2) correctly describe the motion of small heavy particles in a turbulent flow at moderate Reynolds number.

\section{B. Transient regimes and formation of preferential concentrations}

It is well known that inertial particles tend to concentrate into specific regions of the flow. By performing a local analysis of the equations of motion it is possible to show that, for small Stokes numbers, particles heavier than the fluid tend to concentrate onto strain dominated regions of the flow and escape from rotational regions, while the opposite is observed for lighter particles [15, 16, 24]. Hence, particles do not sample uniformly the full velocity field and are sensitive to its geometry.

Being interested in those properties of particle dynamics that are closely linked to the flow geometry at the particle locations (such as the distributions of velocity and of acceleration), one should clearly understand the bias induced by the correlation between particle positions and flow structures. Indeed, highly intermittent spots of the turbulent flow have a strong signature on the acceleration distribution of simple tracers through the phenomenon of vortex trapping 28, 35. Moreover, at variance with tracers, whose dynamics is completely determined by that of the velocity field, inertial particles have their own dynamics. Therefore, for arbitrary initial data, a relaxation time is needed for them to reach a statistically stationary motion. Our simulations are started with a spatially uniform distribution of particles with a vanishing acceleration (velocity equal to that of the fluid). At sufficiently large times, the spatial distribution 

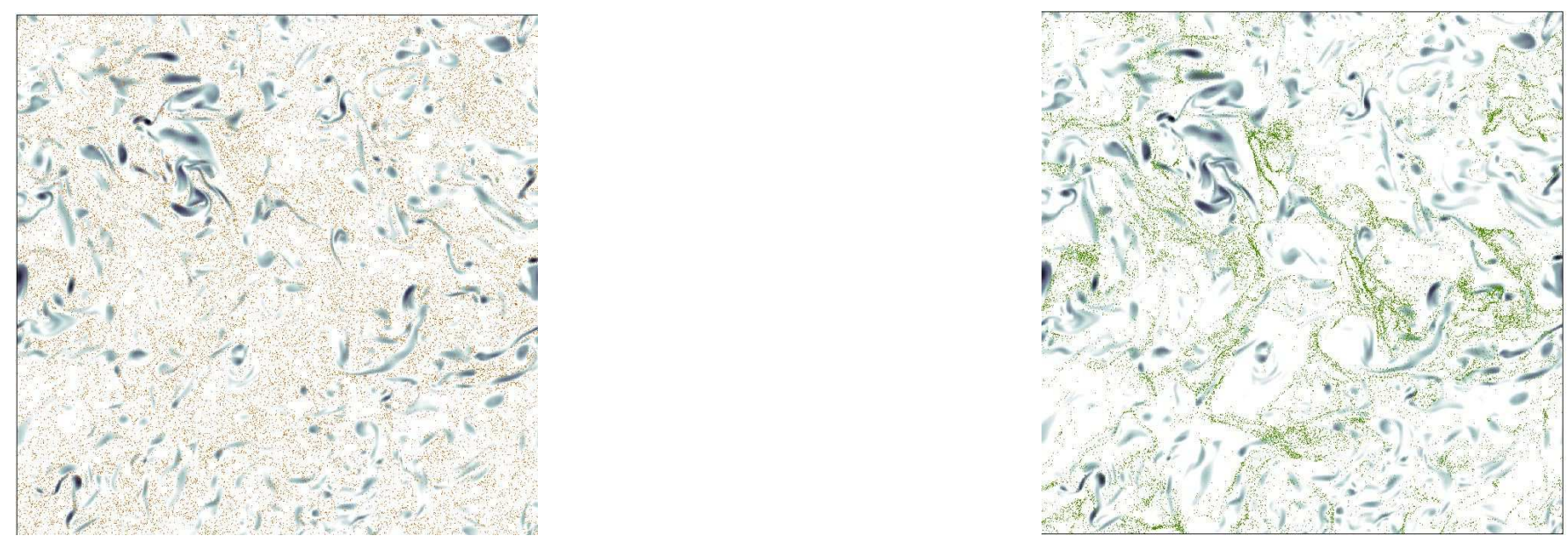

FIG. 2: (Left) For $S t=0.16$, particles positions on a two dimensional slice of the simulation box and, in grey scale, the amplitude of the vorticity field. (Right) The same for $S t=2.03$.

of particles becomes strongly inhomogeneous and strongly correlates with the flow structure (see Fig. 2 for two typical examples of the particle spatial distribution). As one can see, even for small values of the Stokes number, particles get less concentrated in those regions where the flow develops strong vortical structures, represented as dark grey spots on the figure. To have insights into the dynamics of heavy particles and to control the statistical analysis it is important to understand the time scales involved in the dynamical process of formation of such inhomogeneities.

A naive inspection of the equation (2) for the motion of a single particle suggests that after few $\tau_{s}$, the particle velocity should relax to that of the fluid. However this relaxation time is clearly not enough to stabilize the statistical properties of the particle distribution, and in particular to form statistically stationary clusters of particles. Indeed, at least for large $S t$, particles organizes in structures with length scales comparable with the largest ones present in the system (as shown in the right panel of Fig. 2).

To quantify the formation of inhomogeneities in the particle distribution we used two observables. The first one gives a rather global insight and is based on the coarse-grained mass distribution of particles. The second observable is local and is related to the particle distribution at scales of the order of the most violent events of the turbulent flow, namely the fraction of particles in rotation and stretching regions of the flow.

We start the characterization of the inhomogeneous distributions of particles by measuring the degree of concentration

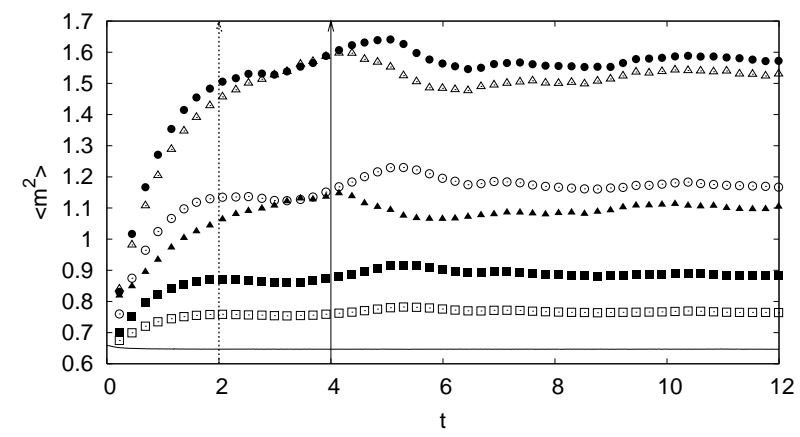

FIG. 3: $\left\langle m^{2}\right\rangle$ vs time, for particles with Stokes numbers $S t=0$ (bottom solid line), $S t=0.16$ (empty boxes), $S t=0.27$ (filled boxes), $S t=0.48$ (empty circles), $S t=0.90$ (filled circles), $S t=1.60$ (empty triangles) and $S t=3.31$ (filled triangles). The two vertical arrows indicate the large scale eddy turnover time $T_{E} \approx 2$. (dashed arrow) and the time chosen to be the end of the transient dynamics $T_{t r} \approx 4$ (solid arrow).

as obtained by coarse-graining the volume in small cubes of side $\Lambda$, and then looking at the distribution of the number $m_{j}$ (or mass) of particles in the $j$-th cell. Figure 3 represents the behavior of the second-order spatial moment $\left\langle m^{2}\right\rangle=(\Lambda / 2 \pi)^{3} \sum_{j} m_{j}^{2}$ of the mass distribution measured on cubes of side $\Lambda=2 \Delta x$. For tracers, which remain uniformly distributed, $\left\langle m^{2}\right\rangle$ stays constant to the initial value, as given by a Poisson distribution. Differently, as soon as $S t>0,\left\langle m^{2}\right\rangle$ starts to increase in time until it stabilizes, fluctuating around a mean value. As shown in the figure 
all curves reach this steady state only for $t \geq 2 \approx T_{E}$, i.e. for times of the order of the large scale eddy turnover time. In performing statistical analysis, for the sake of safety, we consider that the transient ends at $T_{t r} \approx 2 T_{E}$ (second arrow in the figure). Let us notice however that, even in the statistically steady regime, $t>T_{t r}$, fluctuations of $\left\langle m^{2}\right\rangle$ can be rather large and correlated to those of the total energy (see Fig. 4), making the definition of the transition between unsteady and steady regimes somehow difficult. At lower resolutions, we obtained quantitatively similar behaviors. We also checked that the time scale for equilibration does not depend sensibly on $\Lambda$ (this remains true only if $\Lambda$ is not too large).

We now turn to the second observable based on the particle position conditioned on the flow local geometry. There

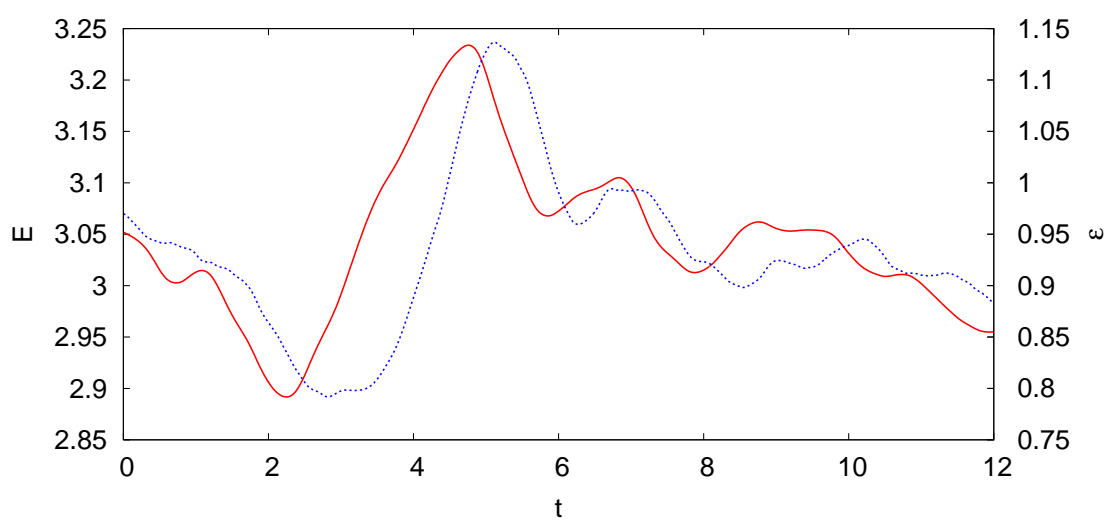

FIG. 4: Time evolution of the total energy $E=\frac{1}{2} \int d r|u(r, t)|^{2}$ (continuous red line) and of the total energy dissipation, $\epsilon$ (dotted blue line).

are several possible ways to identify strain or rotation dominated structures in three dimensions (see Ref. [49] for a review): here we characterize the geometry of the fluid velocity field $u$ by looking at the eigenvalues of the strain matrix $\hat{\sigma}_{i j}=\partial_{i} u_{j}$. Generally, if the eigenvalues are all real, the point is said to be hyperbolic, while if they are all imaginary it is elliptic. In two-dimensions hyperbolic and elliptic points clearly identify strain and vortical structures, respectively. In $3 d$, pure elliptic points do not exist and the identification is less straightforward; the hyperbolic or non-hyperbolic nature of a point can be then identified by looking at the sign of the discriminant [50]:

$$
\Delta=\left(\frac{\operatorname{det}[\hat{\sigma}]}{2}\right)^{2}-\left(\frac{\operatorname{Tr}\left[\hat{\sigma}^{2}\right]}{6}\right)^{3} .
$$

In deriving (66), we omitted the term proportional to $\operatorname{Tr}[\hat{\sigma}]$ because of incompressibility. For $\Delta \leq 0$ the strain matrix has 3 real eigenvalues (strain regions), while for $\Delta>0$ it has one real and 2 complex conjugate eigenvalues (rotational regions). The number of particles $N_{S t}(\Delta>0)$ in $\Delta>0$ regions is represented in Fig. [5 as a function of time and for different Stokes numbers. We chose here to normalize this quantity by the number $N_{S t=0}(\Delta>0)$ of tracers in non-hyperbolic regions to smooth out the instantaneous fluctuations of hyperbolic and non-hyperbolic points in the flow. At $t=0$ this ratio is 1 for all values of $S t$ because particles are uniformly injected in the domain. As time goes on, it follows a very fast drop from the initial value. Unfortunately, the strong fluctuations, correlated to those of the total energy (Fig. (4), do not permit to unambiguously clarify if the time required for $N_{S t}(\Delta>0) / N_{S t=0}(\Delta>0)$ to stabilize is or not of the same order of that needed by $\left\langle m^{2}\right\rangle$. However, according to Fig. [5 it does seem that the correlation with the flow geometry settles on a time scale shorter than that needed to stabilize particles mass moments. It would be interesting to test this point, by studying the simultaneous evolution of particles uniformly injected, and particle already stabilized with the surrounding flow. This would help to disentangle spurious effects induced by the fluctuation of the global properties of the flow.

We conclude this section by commenting the right panel of Fig. [5 where we show the average number of particles normalized by the total number $N_{p},\left\langle N_{S t}(\Delta>0)\right\rangle / N_{p}$ in the stationary regime. As previously observed [43], it appears that preferential concentration is non-monotonic in $S t$, but there is an "optimal" Stokes number for which the effect is maximal, here $S t \simeq 0.55$. This behavior can be understood as follows. To have particles concentrated into specific flow structures, their response time must be fast enough to allow them to follow the flow evolution. Clearly large $S t$ particles are unable to do that and tend to decorrelate from the flow geometry; though their large scale distribution may still be strongly inhomogeneous (as indeed observed, Fig. 21). On the other hand, for $S t \ll 1$ particles behaviour is closer and closer to that of tracers, whose positions do not correlate with the flow geometry. As we will see in next section the balance between these two effects has a strong signature in the probability distribution of particle acceleration. 

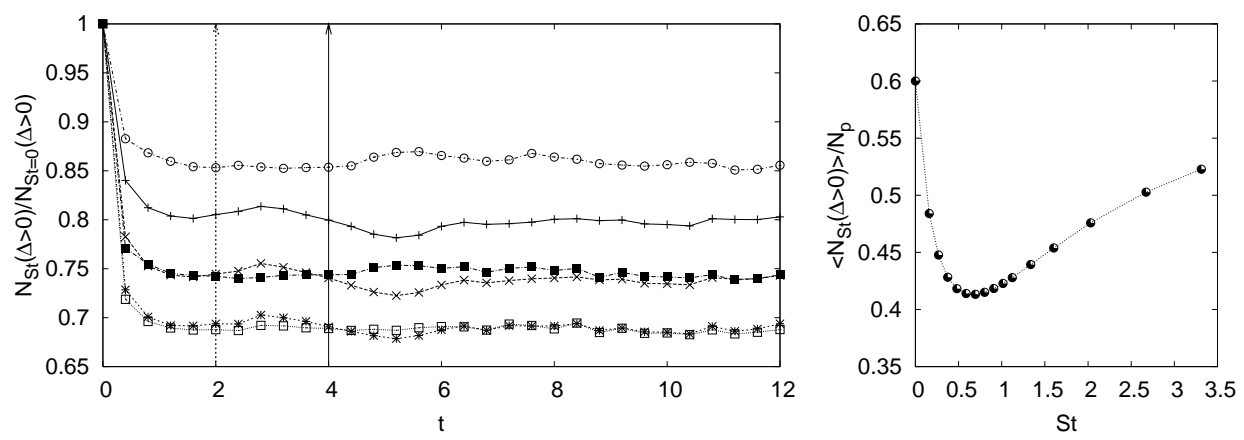

FIG. 5: Average fraction of particles in the non-hyperbolic regions $N_{S t}(\Delta>0) / N_{S t=0}(\Delta>0)$ vs time, for Stokes numbers $S t=0.16$ (plus), $S t=0.27$ (crosses), $S t=0.48$ (stars), $S t=0.90$ (empty boxes), $S t=1.60$ (filled boxes) and $S t=3.31$ (empty circles). The vertical arrows are as in Fig. 3 Right: average fraction of particles in the non-hyperbolic regions $\left\langle N_{S t}(\Delta>0)\right\rangle / N_{p}$ vs $S t$. The average has been performed considering only steady state data, i.e. for $t \geq T_{t r}$.

\section{STATISTICAL PROPERTIES OF THE ACCELERATION}

One of the most striking features that characterize tracers is the very intermittent distribution of the acceleration [28, 29, 31, 35] : fluctuations as strong as 80 times the root mean square acceleration $a_{r m s}$ reflect the tendency of tracers to be trapped into vortical structures 28, 51, 52]. We would like to understand if this property is shared also by inertial particles and what are the main features of the acceleration fluctuations, at changing the importance of inertia. A natural way to proceed is to start from the equations of motion. In the statistically stationary state, the formal solution of Eq.(2) is:

$$
\begin{aligned}
V(t) & =\frac{1}{\tau_{s}} \int_{-\infty}^{t} e^{-(t-s) / \tau_{s}} u(X(s), s) d s \\
a(t) & =\frac{1}{\tau_{s}^{2}} \int_{-\infty}^{t} e^{-(t-s) / \tau_{s}}[u(X(t), t)-u(X(s), s)] d s .
\end{aligned}
$$

It is clear that the kernel $e^{-(t-s) / \tau_{s}}$ acts as a low-pass filter on the fluid velocity differences, suppressing frequencies larger than the inverse of the Stokes time $\tau_{s}^{-1}$. The larger is the Stokes number $S t$, the more important this effect should be. On the other hand, we have indications that the limit of vanishing Stokes number is singular, due to the clusterization of particles onto fractal sets [24, 25]. This should have consequences on the acceleration statistics also. In Fig. [6] the probability density functions for the particles acceleration are plotted for different Stokes numbers and compared with that of tracers. As we could expect, acceleration fluctuations become less and less intermittent as $S t$ increases. To disentangle different effects, it is useful to analyze the two limiting cases of small and large Stokes numbers, separately.

At small St, i.e. $\tau_{s} \ll \tau_{\eta}$, the fluid velocity along the trajectory evolves smoothly in time and the acceleration reduces to $a(t) \simeq d_{t} u(X(t), t)$, i.e. to the derivative of fluid velocity along the inertial particle trajectory. In the limit of vanishing $S t$, this is indistinguishable from the fluid acceleration $D_{t} u(X(t), t)$ evaluated at particle positions and one expects that the particle acceleration essentially coincides with the fluid acceleration. There is however one major difference with the tracer case: inertial particles preferentially concentrate inside regions with low vorticity (see right panel of Fig. [5). In Fig. 7 we plot the results for $a_{\mathrm{rms}}$, as a function of St. Notice the singular effect of the inhomogeneous spatial distribution which results in a drastic reduction of $a_{\mathrm{rms}}$ already for small $S t$. This behavior is even more pronounced for the fourth moment of the acceleration [43]. To better understand the importance of preferential concentration, we have measured the tracers acceleration $\left\langle\left(D_{t} u\right)^{2}\right\rangle^{1 / 2}$ conditioned on the spatial positions of the inertial particles: the result is also plotted in Fig. 7 The agreement of the two curves at small St confirms the validity of the previous argument.

However, at increasing $S t$, the curves start to deviate. While $a_{\mathrm{rms}}$ monotonically decreases in $S t$, the tracer acceleration conditioned on the particle positions has a minimum for $S t \approx 0.5$ close to the maximum of preferential concentration, eventually recovering the value of the unconditioned tracers for larger St. For increasing St, we indeed know that inertial particles explore the small scale structures of the flow more and more homogeneously and a different mechanism is responsible for the reduction of $a_{\mathrm{rms}}$.

At large St, i.e. $\tau_{s} \gg \tau_{\eta}$, we indeed expect the filtering effect to become more and more important. Again, we start 


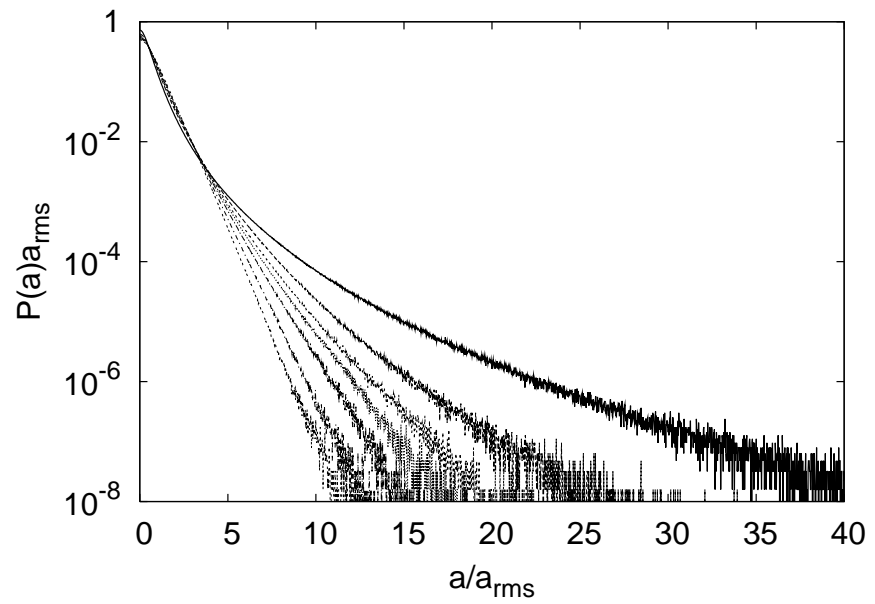

FIG. 6: Normalized acceleration pdf's for a subset of Stokes values ( $S t=0,0.16,0.37,0.58,1.01,2.03,3.31$ from top to bottom) at $R_{\lambda}=185$. The darker line corresponds to tracers, $S t=0$.

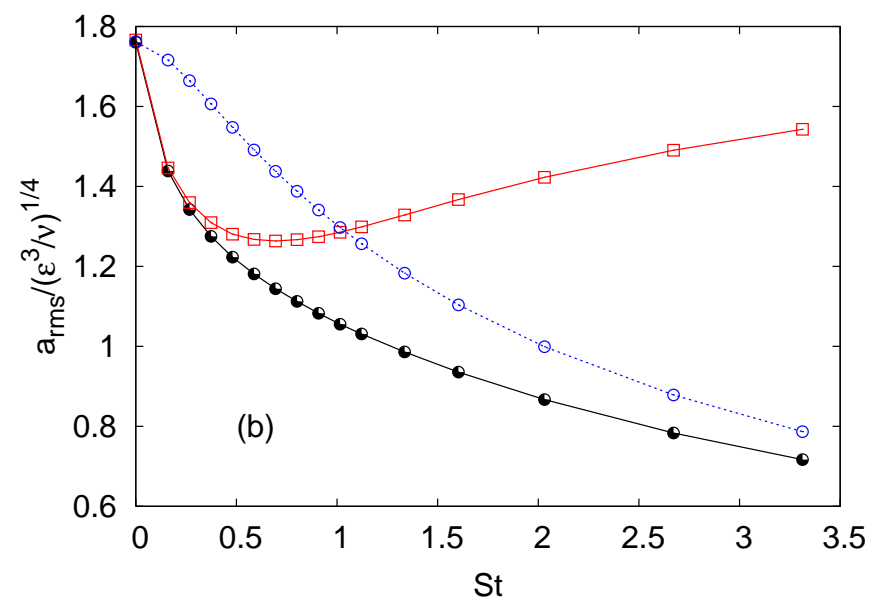

FIG. 7: Comparison between $a_{\mathrm{rms}}$ (fill circles) as a function of Stokes with the acceleration of the fluid tracer conditioned to be on the particle position, $\left\langle\left(D_{t} u\right)^{2}\right\rangle^{1 / 2}$ (empty boxes). The last curve (empty circles), approaching $a_{\mathrm{rms}}$ for large $S t$, is the one obtained from the filtered tracer trajectories, $a_{\mathrm{rms}}^{F}$.

from tracers: for each solution, $x(t)$ of Eq. (3), we define a new velocity, $u^{F}$, obtained from filtering the fluid velocity along the particle trajectory over a time window of the size of the order of the Stokes time:

$$
u^{F}(t)=\frac{1}{\tau_{s}} \int_{-\infty}^{t} e^{-(t-s) / \tau_{s}} u(x(s), s) d s,
$$

then the filtered acceleration is given by $a^{F}=d u^{F} / d t$. In figure 7 the acceleration variance of particles $a_{\mathrm{rms}}$ is compared with that obtained from tracers in the above manner, without any additional spatial conditioning. The curves corresponding to $a_{\mathrm{rms}}$ and to $a_{\mathrm{rms}}^{F}$ become closer and closer as $S t$ increases, supporting the conjecture that preferential concentration for $S t>1$ becomes less important.

For intermediate $S t$ a non trivial interplay between the two above mechanisms takes place. A model, even qualitative, able to bridge the gap between the two limits in still unavailable.

In the limit of small and large St the qualitative trend of the pdf's can be captured by the same arguments as those used for $a_{\text {rms }}$. In Fig. [ 8 we compare the normalized pdf particle acceleration with those obtained by using the tracer acceleration measured on the particle position, $D_{t} u$, and the filtered tracer trajectories, $a^{F}$, respectively. For the smallest Stokes number $S t=0.16$, the overlap between the conditional tracer acceleration and the particle acceleration is almost perfect, while the filtered one is very close to the unconditioned tracer acceleration pdf. The opposite is observed for the largest Stokes number, $S t=3.31$. Notice that the (normalized) conditional tracer acceleration pdf 

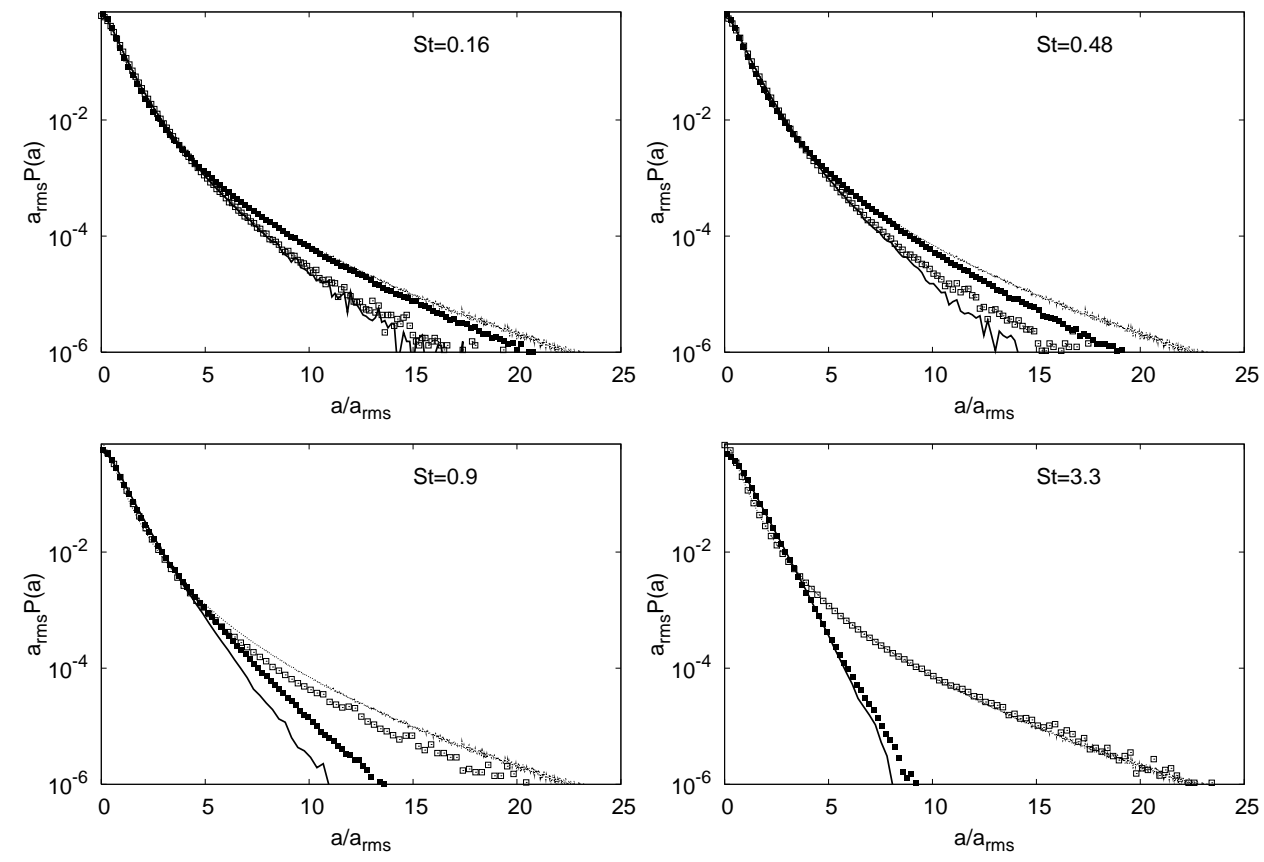

FIG. 8: In each panel the following four curves are shown: the normalized acceleration pdf for inertial particles (solid line), the acceleration pdf of fluid tracers $D_{t} u$ conditioned to particle positions (empty boxes), the acceleration pdf of filtered fluid trajectories $a^{F}$ (filled boxes) and the unconditioned acceleration pdf for fluid tracers(dotted line). Note that the filtered acceleration $a^{F}$ pdf, which is for small $S t$ practically coincident with the (unfiltered and unconditioned) fluid acceleration pdf, becomes closer and closer to the particle acceleration pdf as $S t$ increases. The opposite is observed for the tracer acceleration conditioned to particle positions.

is very close to the unconditioned one, meaning that preferential concentration is no longer effective on the small scale statistics for large $S t$. For two intermediate $S t$ values, we observe the transition from preferential concentration dominated regime to the filtering dominated one.

We conclude this section by going back to the instantaneous distribution of particles for small and large St (see Fig. (2). Both pictures present an inhomogeneous spatial distribution for the inertial particles but for different reasons. At small St (left panel), inhomogeneity is mostly related to the fact that particles escape from large vorticity regions. For such small response times $\tau_{s}$, particles are able to follow the flow variations but the very small scales properties of the fluid are sampled in a biased way. This explains the remarkable agreement between particle acceleration statistics and fluid tracers conditioned on particle positions. At larger Stokes number, the inhomogeneity is less correlated to the fine structures of the flow. Indeed we observe void regions whose typical size can be much larger than the Kolmogorov length scale and comparable to the inertial range scales. In spite of the clear inhomogeneous distribution of particles, the small scales, $r \ll \eta$, are sampled more homogeneously than at smaller $S t$. This is coherent with the fact that the conditional tracer acceleration statistics is very close to the unconditioned one.

\section{ACCELERATION OF POLYDISPERSE SUSPENSIONS}

Suspensions generally involves particles distributed over different sizes. This is true not only for phenomena observed in nature, as for aerosol distribution in the atmosphere, but also for laboratory experiments. In some cases, particles can also considerably change their size with time, as it happens for water droplets in warm clouds [53], so varying their Stokes number. Direct numerical simulations allow to study inertial particles in the idealized case of a given Stokes number, as we have done previously, but also permit to test phenomenological approaches for polydisperse flows. In this section, we study what happens to the acceleration pdf when particles have a non trivial size distribution, or equivalently when the Stokes number of a given suspension is a random variable with a distribution $\mathcal{Q}(S t)$. This is particularly important to have clean and possibly unambiguous comparison of numerical simulations results and laboratory experiments.

To model the acceleration pdf $\mathcal{P}_{\text {poly }}(a)$ in polydisperse flows, we first need a functional form for the pdf, $\mathcal{P}(a \mid S t)$, of particles with a given Stokes number St. Second we make a convolution of the latter distribution with $\mathcal{Q}(S t)$. 


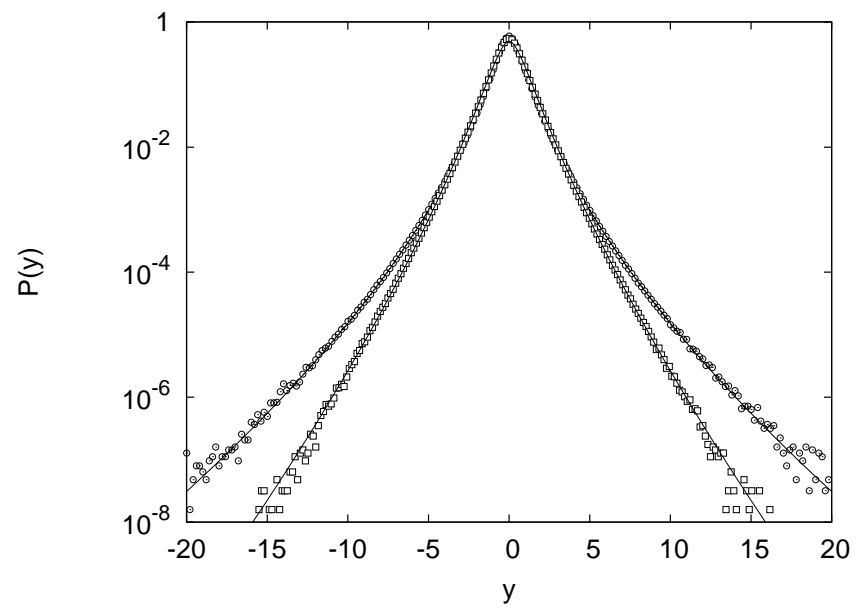

FIG. 9: Comparison of the acceleration pdf from DNS data (symbols) and the fit (solid line) of eq. (9) for two monodisperse cases: for $S t=0.27$ (outer curves) and $S t=1.01$ (inner curves).
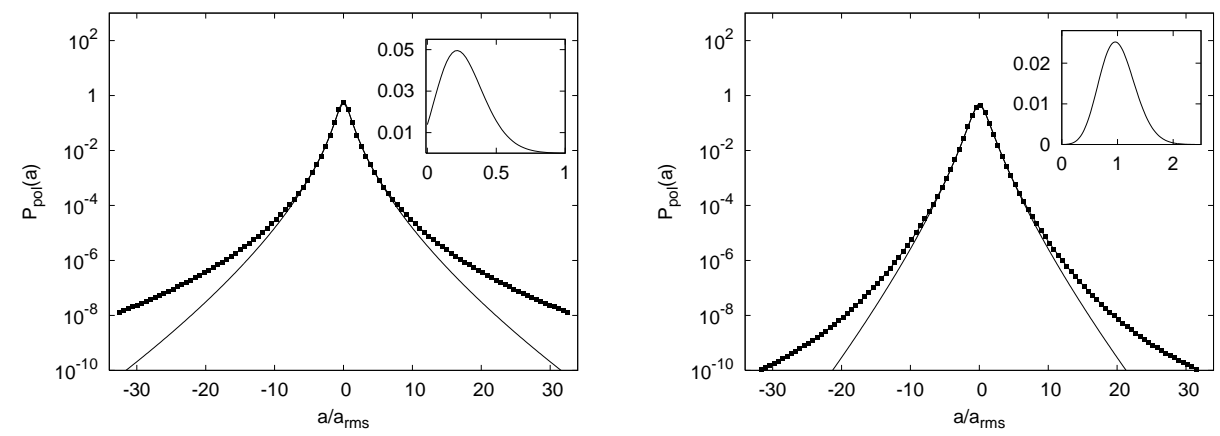

FIG. 10: Comparison between the acceleration pdf for a polydisperse case (filled squares) of mean Stokes value $\overline{S t}$, and a monodisperse case (solid line) at the Stokes number $S t=\overline{S t}$. In the inset, the Poisson distribution $\mathcal{Q}(S t)$ of mean Stokes value $\overline{S t}$. Left panel refers to case with the Stokes number $\overline{S t}=0.27$, while right panel refers to $\overline{S t}=1.01$.

Building up a phenomenological model for the acceleration pdf of monodisperse suspension valid at different Stokes number is not an easy goal. For tracers, the multifractal formalism, without free parameter, is able to predict an acceleration pdf which is in excellent agreement with DNS data [36]. However any simple extension of such approach to heavy particles does not seem to work, because of the almost singular nature of the small St limit and of the complex interplay between preferential concentration and time filtering. In the lack of such a model, the first step is done by fitting the acceleration pdf at changing the Stokes number. At small values of the acceleration, i.e. in the pdf core, we expect to have a Gaussian-like profile $\mathcal{P}(a \mid S t) \sim \exp \left(-a^{2}\right)$; while for large fluctuations $a \gg a_{r m s}$, we should recover a distribution with stretched exponential tails, $\mathcal{P}(a \mid S t) \propto \exp \left(-a^{\gamma}\right)$. A simple functional form for the pdf, $\mathcal{P}(a \mid S t)$ of the normalized acceleration $y=a / a_{r m s}(S t)$ satisfying these two requirements is:

$$
\tilde{\mathcal{P}}(y \mid S t) \equiv a_{r m s} \mathcal{P}\left(y a_{r m s} \mid S t\right)=\exp \left\{-\frac{c_{1}|y|^{2}}{1+c_{3} y^{c_{2}}}+c_{4}\right\} .
$$

Here, to have a simple fitting procedure, we have used four free parameters $c_{i}(S t)$ with $i=[1, \ldots, 4]$, although two of them may be fixed by the requirement of having the pdf normalized to unit area and unit variance. Of course, the most important parameter is $c_{2}(S t)$ which fixes the Stokes number dependency of the far tail exponent $\gamma=2-c_{2}$.

The acceleration pdf measured in our DNS has been fitted with expression (9) for all available St. The values for the four free parameters are those reported in Table VI They all gently, but non monotonically, vary with the Stokes number. In particular, the exponent $c_{2}$, which controls the fat tails, goes from about $\sim 1.6$, for the smallest Stokes, to $\sim 1.2$, for the largest available one. This is in agreement with the tendency towards a less intermittent distribution for particles with a larger inertia. From Fig. 9] we can see that the fitting formula (9) reproduces well the acceleration distribution (here shown for $S t=0.16$ and $S t=1.01$ only).

To assess the variations for a polydisperse suspension, we make the convolution between the acceleration pdf condi- 


\begin{tabular}{ccccccccccccccccccc}
\hline St & 0 & 0.16 & 0.27 & 0.37 & 0.48 & 0.59 & 0.69 & 0.80 & & St & 0.91 & 1.01 & 1.12 & 1.34 & 1.60 & 2.03 & 2.67 & 3.31 \\
\hline$c_{1}$ & 2.68 & 2.17 & 2.24 & 2.31 & 1.67 & 1.64 & 1.83 & 1.66 & & $c_{1}$ & 0.83 & 1.64 & 1.15 & 1.08 & 1.18 & 1.95 & 0.89 & 0.76 \\
$c_{2}$ & 1.55 & 1.43 & 1.38 & 1.35 & 1.36 & 1.33 & 1.29 & 1.28 & & $c_{2}$ & 1.40 & 1.25 & 1.30 & 1.28 & 1.24 & 1.11 & 1.23 & 1.27 \\
$c_{3}$ & 0.79 & 0.75 & 0.83 & 0.91 & 0.62 & 0.63 & 0.76 & 0.68 & & $c_{3}$ & 0.24 & 0.69 & 0.42 & 0.39 & 0.46 & 1.00 & 0.30 & 0.21 \\
$c_{4}$ & -0.41 & -0.53 & -0.53 & -0.53 & -0.65 & -0.67 & -0.64 & -0.68 & & $c_{4}$ & -0.98 & -0.69 & -0.82 & -0.85 & -0.81 & -0.67 & -0.90 & -0.94 \\
\hline
\end{tabular}

TABLE II: The values of the fitting parameters $c_{i}$ at changing the Stokes number. Notice that only two out of the four parameters are indeed independent because the pdf must be normalized to have unit area and unit variance. The fitted functional form is not meant to have a solid phenomenological base. It is used, as simple as it is, to estimate the importance of polydisperse suspensions with respect to monodysperse ones.

tioned on the Stokes number $\mathcal{P}(a \mid S t)$ and the Stokes number distribution $\mathcal{Q}(S t)$ :

$$
\mathcal{P}_{\text {pol }}(a)=\int d S t \mathcal{P}(a \mid S t) \mathcal{Q}(S t) .
$$

A possible choice for $\mathcal{Q}(S t)$ is a Poisson-like distribution: for any available Stokes number $S t$, we generate a Poisson pdf with mean value $\overline{S t}=S t$ and we insert it as test curve to evaluate the convolution (10). In Fig. [10 we compare the polydisperse pdf (10) obtained with a Poissonian distribution, $\mathcal{Q}(S t)$, with a given $\overline{S t}$ and a monodisperse distribution with $S t=\overline{S t}$. We do it for two different average Stokes number, namely $\overline{S t}=0.27,1.01$.

As expected, the effect of a random distribution for the Stokes number is much more important for the tails than for the cores. We recall also that, in the case of monodisperse suspensions the pdf tails are the most sensitive to $S t$ variations. Hence, any polydisperse suspensions characterized by a particle size distribution skewed towards small values of the Stoeks numeber should develop more intermittent tails than those expected on the basis of their mean Stokes value.

\section{CONCLUSIONS AND PERSPECTIVES}

Direct numerical simulations, even at moderate Reynolds number, represent a valuable tool for the study of the Lagrangian motion of heavy particles. Here we have described the specific set up of our numerical study, analyzed the importance of preferential concentration and particle clustering as a function of the Stokes number. Moreover, we have explored the transient dynamics which precedes the settling of the particle motion onto a statistically steady state in terms of the particle distribution. This may be particularly important for real observations and laboratory experiments, where long time records of particle motion are hardly attainable. We have also reviewed and extended some recent results about the acceleration pdf of inertial particles, at varying the importance of inertia. The main conclusions are (i) preferential concentration plays an almost singular role at small Stokes, since even a small inertia is sufficient to expel particles from vortical regions where the strongest acceleration fluctuations are experienced; (ii) for small Stokes, a good quantitative agreement between the inertial particle acceleration and the conditioned fluid tracer acceleration is obtained; (iii) at large Stokes, the main effects is filtering of the velocity induced by the response Stokes times.

The formulation of a phenomenological model able to describe the inertial particle acceleration as a function of both Stokes and Reynolds numbers is still beyond our reach. An easy fitting procedure has however been proposed for polydisperse suspensions, the most common experimental situation. For polydisperse cases, we show that the simple approach of characterizing the suspension in terms of its average Stokes number without taking into account the Stokes number distribution can give a systematic bias, particularly if the particle distribution is skewed towards small Stokes values. It would be extremely important to test the proposed approach with experimental data. Finally it would be interesting to numerically investigate $S t \ll 1$ particles to get a better understanding of the almost singular behavior that has been observed for the acceleration.

We acknowledge useful discussions with S. Ayyalasomayajula, E. Bodenschatz, A. Gylfason, G. Falkovich and Z. Warhaft. This work has been partially supported by the EU under the research training network HPRN-CT-200200300 "Stirring and Mixing". Numerical simulations have been performed thanks to the support of CINECA (Italy) and IDRIS (France) under the HPC Europa project (RII3-CT-2003-506079). We thank also the "Centro Ricerche e 
Studi Enrico Fermi" and N. Tantalo for technical support.

[1] Eaton, J.K.aqnd Fessler, J.R. 1994 Preferential concentrations of particles by turbulence. Int. J. Multiphase Flow 20, 169-209.

[2] Pinsky, M. and Khain, A. 1997 Turbulence effects on droplet growth and size distribution in clouds-a review. J. Aerosol Sci. 28, 1177-1214.

[3] Falkovich, G. Fouxon, A. and Stepanov, M. 2002 Acceleration of rain initiation by cloud turbulence. Nature 419, 151-154.

[4] Shaw, R.A. 2003 Particle-turbulence interactions in atmospheric clouds. Ann. Rev. Fluid Mech. 35, 183-227.

[5] Sundaram S. and Collins, L.R. 1997 Collision statistics in an isotropic particle-laden turbulent suspension. Part 1. Direct numerical simulations. J. Fluid Mech. 335, 75-109.

[6] Rothschild, B.J. and Osborn, T.R. 1988 Small-scale turbulence and plankton contact rates. J. Plankton Res. 10, 465-474.

[7] Lewis, D. and Pedley, T. 2000 Planktonic contact rates in homogeneous isotropic turbulence: Theoretical predictions and kinematic simulations. J. Theor. Biol. 205, 377-408.

[8] Post, S. and Abraham, J. 2002 Modeling the outcome of drop-drop collisions in Diesel sprays. Int. J. of Multiphase Flow 28, 997-1019.

[9] Villedieu P. and Hylkema J. 2000 Modèles numériques lagrangiens pour la phase dispersée dans les propulseurs à poudre. Rapport technique ONERA.

[10] Csanady, G. 1980 Turbulent diffusion in the environment. Geophysics and Astrophysics Monographs Vol. 3 D. Reidel Publishing Company.

[11] Seinfeld J. 1986 Atmospheric chemistry and physics of air pollution. J. Wiley and Sons.

[12] Balkovsky, E. Falkovich, G. and Fouxon, A. 2001 Intermittent distribution of inertial particles in turbulent flows. Phys. Rev. Lett. 86, 2790-2793.

[13] Falkovich, G. and Pumir, A. 2004 Intermittent distribution of heavy particles in a turbulent flow. Phys. Fluids 16, L47-L51.

[14] Zaichik, L.I. Simonin, O. and Alipchenkov V.M. 2003 Two statistical models for predicting collision rates of inertial particles in homogeneous isotropic turbulence. Phys. Fluids 15, 2995-3005.

[15] Elperin, T. Kleeorin, N. Liberman, M.A. L'vov, V. S. Pomyalov, A. and Rogachevskii, I. 2003 Clustering of inertial particles in turbulents flows. e-arXive nlin.CD/0305017

[16] Squires, K.D. and Eaton, J.K. 1991 Preferential concentration of particles by turbulence. Phys. Fluids A 3, 1169-1178.

[17] Boivin, M. Simonin, O. and Squires, KD. 1998 Direct numerical simulation of turbulence modulation by particles in isotropic turbulence. J. Fluid Mech. 375, 235-263.

[18] Reade, W.C. and Collins, L.R. 2000 A numerical study of the particle size distribution of an aerosol undergoing turbulent coagulation. J. Fluid Mech. 415, 45-64.

[19] Zhou, Y. Wexler, A. and Wang, L.-P. 2001 Modelling turbulent collision of bidisperse inertial particles. J. Fluid Mech. 433, $77-104$.

[20] Chun, J. Koch, D.L. Rani, S. Ahluwalia, A. and Collins, L.R. 2005 Clustering of aerosol particles in isotropic turbulence. J. Fluid Mech. 536, 219-251.

[21] Collins, L.R. and Keswani, A. 2004 Reynolds number scaling of particle clustering in turbulent aerosols. New J. Phys. 6, 119.

[22] Sigurgeirsson, H. and Stuart, A.M. 2002 A model for preferential concentration. Phys. Fluids 14 $4352-4361$.

[23] Bec, J. Gawedzki, K. and Horvai, P. 2004 Multifractal clustering in compressible flows. Phys. Rev. Lett. 92, 224501.

[24] Bec, J. 2005 Multifractal concentrations of inertial particles in smooth random flows. J. Fluid Mech. 528, $255-277$.

[25] Bec, J. Celani, A. Cencini, M. and Musacchio, S. 2005 Clustering and collisions of heavy particles in random smooth flows. Phys. Fluids 17, 073301.

[26] Boffetta, G. De Lillo, F. and Gamba, A. 2004 Large scale inhomogeneity of inertial particles in turbulent flows. Phys. Fluids 16, L20-L24.

[27] Warhaft, Z. Gylfason, A. and Ayyalasomayajula, S. 2005 private communication.

[28] La Porta, A. Voth, G.A. Crawford, A.M. Alexander, J. and Bodenschatz, E. 2001 Fluid particle accelerations in fully developed turbulence. Nature 409, 1017-1019.

[29] La Porta, A. Voth, G.A. Crawford, A.M. Alexander, J. and Bodenschatz, E. 2002 Measurement of particle accelerations in fully developed turbulence. J. Fluid Mech. 469, 121-160.

[30] Ott, S. and Mann, J. 2000 An experimental investigation of relative diffusion of particle pairs in three- dimensional turbulent flow. J. Fluid Mech. 422, 207-223.

[31] Mordant, N. Metz, P. Michel, O. and Pinton, J.-F. 2001 Measurement of Lagrangian velocity in fully developed turbulence. Phys. Rev. Lett. 87, 214501.

[32] Mordant, N. Lévêque, E. and Pinton, J.-F. 2004 Experimental and numerical study of the Lagrangian dynamics of high Reynolds turbulence. New Journal of Physics 6, 116.

[33] Sawford, B.L. and Guest, F.M. 1991 Lagrangian statical simulation of the turbulent motion of heavy particles. BoundaryLayer Meteorol. 54, 147-166.

[34] Sawford, B.L. Yeung, P.K. Borgas, M.S. Vedula, P. La Porta, A. Crawford, A.M. Bodenschatz, E. 2003 Conditional and unconditional acceleration statistics in turbulence. Phys. Fluids 15, 3478-3489. 
[35] Biferale, L. Boffetta, G. Celani, A. Lanotte, A. and Toschi, F. 2005 Particle trapping in three-dimensional fully developed turbulence. Phys. Fluids. 17, 021701

[36] Biferale, L. Boffetta, G. Celani, A. Devenish, B.J. Lanotte, A. and Toschi, F. 2004 Multifractal statistics of Lagrangian velocity and acceleration in turbulence. Phys. Rev. Lett. 93, 064502.

[37] Yeung, P.K. 2002 Lagrangian Investigation of Turbulence Ann. Rev. Fluid Mech. 34, 115.

[38] Yeung, P.K. 2001 Lagrangian characteristics of turbulence and scalar transport in direct numerical simulations, J. Fluid Mech. 427, 241-274.

[39] Ishihara, T. and Kaneda, Y. 2002 Relative diffusion of a pair of fluid particles in the inertial subrange of turbulence Phys. Fluids 14, L69.

[40] Gotoh, T. and Fukuyama, D. 2001 Pressure Spectrum in Homogeneous Turbulence Phys. Rev. Lett. 86, 3775-3778.

[41] Hill, R.J. 2002 Scaling of acceleration in locally isotropic turbulence. J. Fluid Mech. 452, 361-370.

[42] Gotoh, T. and Kraichnan, R.H. 2004 Turbulence and Tsallis Statistics Physica D 193, 231-244

[43] Bec, J. Biferale, L. Boffetta, G. Celani, A. Cencini, M. Lanotte, A. Musacchio, S. and Toschi,F. 2006 Acceleration statistics of heavy particles in turbulence. to appear on J. Fluid Mech..

[44] Maxey, M.R. and Riley, J. 1983 Equation of motion of a small rigid sphere in a nonuniform flow. Phys. Fluids 26, 883-889.

[45] Maxey, M.R. 1987. The gravitational settling of aerosol particles in homogeneous turbulence and random flow fields. $J$. Fluid Mech. 174, 441-465.

[46] Gatignol, R. 1983 The Faxen formulae for a rigid particle in an unsteady non-uniform Stokes flow. J. Mec. Theor. Appl. $\mathbf{1}, 143$.

[47] Generally, these hypothesis are also sufficient to neglect the contributions of the Basset history and the added mass terms, and the Faxén corrections.

[48] Chen, S. Doolen, G.D. Kraichnan, R.H. and She, Z.S. 1993 On statistical correlations between velocity increments and locally averaged dissipation in homogeneous turbulence. Phys. Fluids A 5, 458-463.

[49] Haller, G 2005 An objective definition of a vortex J. Fluid Mech. 525, 1-26.

[50] Chong, M.S., Perry, A.E. and Cantwell, B.J. 1990 A general classification of three-dimensional flow field. Phys. Fluids A 2, 765-777.

[51] Biferale, L. and Toschi, F. 2006. Joint statistics of acceleration and vorticity in fully developed turbulence Journ. of Turbulence to appear.

[52] Toschi, F. Biferale, L Boffetta, G. Celani, A. Devenish, B. and Lanotte, A. 2006 Acceleration and vortex filaments in turbulence. Journ. of Turbulence to appear.

[53] Pruppacher, H.R. and Klett, J.D. 1997 Microphysics of Clouds and Precipitation. Kluwer Acad. Publisher. 